\title{
Benefit of Whole Pelvic Radiotherapy Combined with Neoadjuvant Androgen Deprivation for the High-Risk Prostate Cancer
}

\author{
Piotr Milecki, ${ }^{1}$ Maciej Baczyk, ${ }^{2}$ Janusz Skowronek, ${ }^{3}$ Andrzej Antczak, ${ }^{4}$ \\ Zbigniew Kwias, ${ }^{4}$ and Piotr Martenka ${ }^{1}$
}

${ }^{1}$ Department of Radiotherapy, Greater Poland Cancer Centre, Poznan 61-866, Poland

${ }^{2}$ Department of Nuclear Medicine, Medical University, Poznań 60-355, Poland

${ }^{3}$ Department of Brachytherapy, Greater Poland Cancer Centre, Poznań 61-866, Poland

${ }^{4}$ Faculty of Urology, Medical University, Poznań 61-285, Poland

Correspondence should be addressed to Piotr Milecki, piotr.milecki@wco.pl

Received 22 March 2009; Accepted 27 July 2009

Recommended by Daehee Kang

Aim. To study whether use of neoadjuvant androgen deprivation therapy (N-ADT) combined with whole pelvic radiotherapy (WPRT) for high-risk prostate cancer patients was associated with survival benefit over prostate radiotherapy (PORT) only. Material and Methods. Between 1999 and 2004, 162 high-risk prostate cancer patients were treated with radiotherapy combined with long-term androgen deprivation therapy (L-ADT). Patients were prospectively assigned into two groups: A (N-ADT + WPRT $+\mathrm{L}-\mathrm{ADT}) n=70$ pts, B (PORT $+\mathrm{L}-\mathrm{ADT}) n=92$ pts. Results. The 5 -year actuarial overall survival (OS) rates were $89 \%$ for A and $78 \%$ for $\mathrm{B}(P=.13)$. The 5 -year actuarial cause specific survival $(\mathrm{CSS})$ rates were $\mathrm{A}=90 \%$ and $\mathrm{B}=79 \%(P=.01)$. Biochemical progression-free survival (bPFS) rates were $52 \%$ versus $40 \%(P=.07)$, for groups A and $\mathrm{B}$, respectively. Conclusions. The WPRT combined with N-ADT compared to PORT for high-risk patients resulted in improvement in CSS and bPFS; however no OS benefit was observed.

Copyright () 2009 Piotr Milecki et al. This is an open access article distributed under the Creative Commons Attribution License, which permits unrestricted use, distribution, and reproduction in any medium, provided the original work is properly cited.

\section{Introduction}

The number of diagnosed new cases of prostate cancer is increasing in the United States as well in the European Union [1-3]. Additionally, for the last decades significant migration of clinical stages has been observed which resulted in rise in the number of patients in low or intermediate risk of relapse [4]. On the other hand, the number of patients representing an advanced stage of disease, which is related to the high-risk of relapse, is decreasing. Despite these facts, a significant number of patients constitute the high-risk group with high probability of micrometastases in the regional lymph nodes. For this group optimal management remains controversial. The role of radiotherapy (RT) in treatment of high-risk prostate cancer is generally established, although there are still many controversies concerning the optimal total dose, definition of irradiated volume (whole pelvis versus prostate only), and ways of the most efficient combination with androgen deprivation therapy (neoadjuvant versus adjuvant and the timing of androgen deprivation) [5]. Several clinical studies have demonstrated that RT combined with adjuvant long-term androgen deprivation therapy (L-ADT) for high-risk patients is associated with improved biochemical progression-free survival (bPFS), cause-specific survival (CSS), and overall survival (OS) [69]. However, these studies did not compare the whole pelvic radiotherapy (WPRT) combined with neoadjuvant androgen deprivation therapy (N-ADT) and L-ADT with prostate radiotherapy only (PORT) combined with L-ADT $[10,11]$. In consequence, the effectiveness of the regional lymphatic network irradiation combined with $\mathrm{N}$-ADT has not been established. It is extremely important to evaluate the role of WPRT because pelvic irradiation could raise the intensity of side effects $[12,13]$. Thus, the question arises about the 
therapeutic gain related to WPRT combined with N-ADT over the prostate only irradiation [14-17].

\section{Purpose}

The aim of this study was to evaluate whether N-ADT combined with WPRT and L-ADT is associated with survival benefit over PORT and L-ADT for the cohort of patients defined as the high-risk group of prostate cancer.

\section{Materials and Methods}

3.1. Patient Characteristics. From May 1999 to December 2004, 162 patients were entered into prospective comparison study. All patients defined as the high risk were treated with three-dimensional conformal radiotherapy (3D CRT) and L-ADT at the Greater Poland Cancer Center in Poznan, Poland. All patients represented good general performance status defined as 0 or 1 according to the Eastern Cooperative Oncology Group (ECOG) classification. All patients had a histological diagnosis of adenocarcinoma classified according to Gleason score. Patients were classified as the high-risk patients when at least one of the following conditions was present: Gleason score $>7$ or initial prostate-specific antigen (PSA) level $>20 \mathrm{ng} / \mathrm{mL}$ or T3. Patient's characteristics are presented in Table 1.

Statistic analysis was conducted to explore the balance of known prognostic factors in the two groups. The distribution of age, Gleason score, PSA, and risk of lymph nodes metastases was similar between the two groups (Table 1).

The average level of PSA before the beginning of treatment was for the group A $37.3 \mathrm{ng} / \mathrm{mL}$ (range: 19$70 \mathrm{ng} / \mathrm{mL}$ ) and for the group B $38.1 \mathrm{ng} / \mathrm{mL}$ (range: 6$70 \mathrm{ng} / \mathrm{mL}$ ). All patients before starting treatment had bone scan and chest X-ray. Pelvic lymph nodes were evaluated by computer tomography. Only patients with no signs of metastases in these examinations were treated with radical intent. We did not perform diagnostic lymphadenectomy of the pelvic lymph nodes. The median level of risk metastases to lymph nodes based on the formula proposed by Roach III et al. for all patients was 32\% (range: 23\%-67\%) [18]. Treatment strategy (N-ADT + WPRT versus PORT) for individual patient was chosen after discussing all cons and pros of two methods with treating physicians (urologist and radiation oncologist). As many as 70 out of $162(43 \%)$ patients chose WPRT with N-ADT (group A). The second option of treatment (PORT + L-ADT) was chosen by 92 (57\%) of 162 patients (group B).

3.2. Androgen Deprivation Therapy (ADT). All patients included into group A have started N-ADT immediately after confirmation of histological diagnosis. All patients were treated with LHRH analogs started on a 1 monthly preparation of gosereline acetate and then moved to 3monthly $10.8 \mathrm{mg}$ depot preparation. Additionally, patients received two weeks before LHRH analog treatment a shorttime antiandrogen therapy to prevent the flare syndrome (flutamide $250 \mathrm{mg}$ p.o. tid for 4 weeks). N-ADT duration ranged from 2 to 10 months (median 4.4 months). Androgen deprivation therapy was also continued during the course of RT. All patients (group A and group B) were treated with L-ADT (LHRH analog) from the last day of irradiation. The median duration of L-ADT was 28.4 months (range: 3-37) and 29.1 months (range: 9-39) for the group A and group B, respectively. In case of biochemical failure (the PSA recurrence defined according to the Phoenix definition) [19] an antiandrogen was added to LHRH analog as a second line hormonal therapy or the LHRH analog was introduced again.

3.3. Radiotherapy Technique. Simulation and treatment were performed in a supine position (using knee support and laser beams) with "comfortable" full bladder filling. For radiotherapy planning all patients underwent pelvic CT scan in the treatment position; $5-\mathrm{mm}$ slice thickness is used from the top of iliac bone to at least $5 \mathrm{~cm}$ below the base of the penis. For all patients the following organs at risk (OAR) were outlined: bladder, rectum, intestine, and femoral heads. Planning target volume 2 (PTV 2) was the pelvic lymph nodes (the obturator, hypogastric, presacral, internal, and external iliac nodes) and prostate with seminal vesicles with a $1 \mathrm{~cm}$ margin and was treated with total dose of $46 \mathrm{~Gy}$. Therefore, the superior border of the field was placed at the level of division of common iliac vessels. PTV 1 encompassed the prostate and seminal vesicles with $1 \mathrm{~cm}$ margin, except posterior part of PTV 1 where $0.5 \mathrm{~cm}$ margin was added.

The reproducibility of the irradiated fields was checked by electronic portal imaging device (EPID) performed at the start of the treatment (the first day of irradiation) and weakly thereafter or when the treatment phase was changed. In vivo dosimetry based on termoluminescence dosimetry (TLD) was performed for each patient at the beginning (usually at the first day of irradiation) and in the middle of the treatment. Radiotherapy was administered using $20 \mathrm{MV}$ photon beam (Clinac $2300 \mathrm{CD}$, Varian) in a daily fraction of $1.8 \mathrm{~Gy}$ or $2.0 \mathrm{~Gy}$ for the total median dose of $70.2 \mathrm{~Gy}$ (range: $66 \mathrm{~Gy}-74 \mathrm{~Gy}$ ). In each case 3D CRT was based on 3-field or 4-field techniques with the application of the multileaf collimators [20]. For the subgroup of patients irradiated to pelvic the total median dose was 46.4 Gy (range: $44 \mathrm{~Gy}-50 \mathrm{~Gy}$ ) which was applied with box technique (4-field technique).

Acute side effects were evaluated during the irradiation course and than 1 month after the completion of treatment using the Radiation Therapy Oncology Group (RTOG)/the European Organization on Radiation Therapy Criteria (EORTC) morbidity scoring scale [21]. Late side effects were evaluated according to the RTOG/EORTC scale during each visit.

3.4. Followup. Patients were seen one month after the completion of radiotherapy and every 3 months thereafter. Patients alternated their appointments between their urologists and radiation oncologists. During each visit serum PSA level was determined and additionally digital rectal examination (DRE) and clinical evaluation were performed. The Tandem-R monoclonal method with detection sensitivity of $0.02 \mathrm{ng} / \mathrm{mL}$ was used for PSA measurement. The median 
TABLe 1: Patient's characteristics entered into prospective study for high-risk patients $(n=162)$.

\begin{tabular}{|c|c|c|c|}
\hline Characteristic & WPRT + N-ADT (group A) & PORT (group B) & $P^{*}$ \\
\hline Patients $(n)$ & $70(100 \%)$ & $92(100 \%)$ & - \\
\hline Mean age (years) & 68.1 (range: $47-78$ ) & 67.9 (range: 48-79) & .81 \\
\hline \multicolumn{4}{|l|}{ Gleason score } \\
\hline $2-7$ & $34(49 \%)$ & $50(55 \%)$ & \multirow{2}{*}{.56} \\
\hline $8-10$ & $36(51 \%)$ & $42(45 \%)$ & \\
\hline \multicolumn{4}{|l|}{ Clinical stage } \\
\hline $\mathrm{T} 2 \mathrm{c}$ & $20(29 \%)$ & $44(48 \%)$ & \multirow{2}{*}{.005} \\
\hline $\mathrm{T} 3$ & $50(71 \%)$ & $48(52 \%)$ & \\
\hline Total dose & $70.1 \mathrm{~Gy}$ & $70.3 \mathrm{~Gy}$ & .22 \\
\hline \multicolumn{4}{|l|}{ PSA (ng/mL) } \\
\hline$<30$ & $25(36 \%)$ & $41(44 \%)$ & \multirow{2}{*}{.32} \\
\hline$>30$ & $45(64 \%)$ & $51(56 \%)$ & \\
\hline \multicolumn{4}{|l|}{$\% N+$} \\
\hline$<30 \%$ & $19(27 \%)$ & $38(41 \%)$ & \multirow{2}{*}{.088} \\
\hline$>30 \%$ & $51(73 \%)$ & $54(59 \%)$ & \\
\hline Duration of L-ADT (months) & 28.4 & 29.1 & .13 \\
\hline Median followup (months) & 54 & 55 & .21 \\
\hline
\end{tabular}

followup duration was 54 months (range: 6-101 months) and 55 months (range: 7-99 months) for groups A and B, respectively.

3.5. Statistics. Primary endpoints for analysis were overall survival (OS), cause specific survival (CSS), distant metastases-free survival (DMFS), and biochemical progression-free survival (bPFS). OS reflected all deaths, CSS reported deaths that could be attributed to prostate cancer. In those cases for which the cause of death was unclear, death was considered as a result of prostate cancer in case when clinically evident prostate cancer was present at the time of death.

The biochemical progression endpoint (PSA) for which outcome was compared was based on the recommendations of the RTOG-ASTRO Phoenix consensus [19]. This definition was selected because it is more accurate than widely used ASTRO definition [22], especially for patients treated with $\mathrm{RT}$ and ADT.

Actuarial results for OS, CSS, DMFS, bPFS, and for late morbidities rates were calculated using the KaplanMeier method. The log rank test was performed to find the correlations between the actuarial results of treatment for the following clinical parameters: age, T stage, Gleason score, PSA, WPRT + N-ADT, PORT. For these calculations $P$-value of $\leq .05$ was considered as statistically significant.

\section{Results}

The 5 -year actuarial OS rates were $89 \%$ and $78 \%$ for group A (N-ADT + WPRT) and group B (PORT), respectively

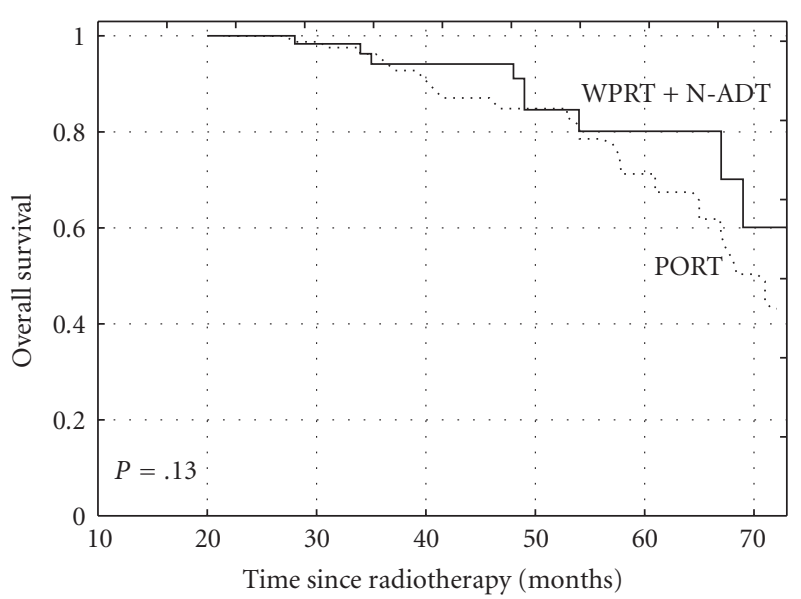

FIgURE 1: The Kaplan-Meier curves comparing overall survival (OS) for patients at high risk of nodal involvement, as defined in text receiving whole pelvic radiotherapy (WPRT) combined with neoadjuvant androgen deprivation therapy $(\mathrm{N}-\mathrm{ADT})$ or prostate radiotherapy only (PORT), $(P=.13)$.

(log rank test, $P=.13$ ) (Figure 1). The 5-year actuarial CSS rates were $90 \%$ and $79 \%$ for the groups A and B, respectively (long rank test, $P=.01$ ) (Figure 2). The 5-year bPFS rates were $52 \%$ versus $40 \%(P=.07)$, for groups $\mathrm{A}$ and B, respectively (Figure 3). Distant metastases-free survival (DMFS) for patients at high-risk of nodal involvement, as defined in text, was statistically significantly better for patients treated in combined arm $(P=.04)$ (Figure 4). 


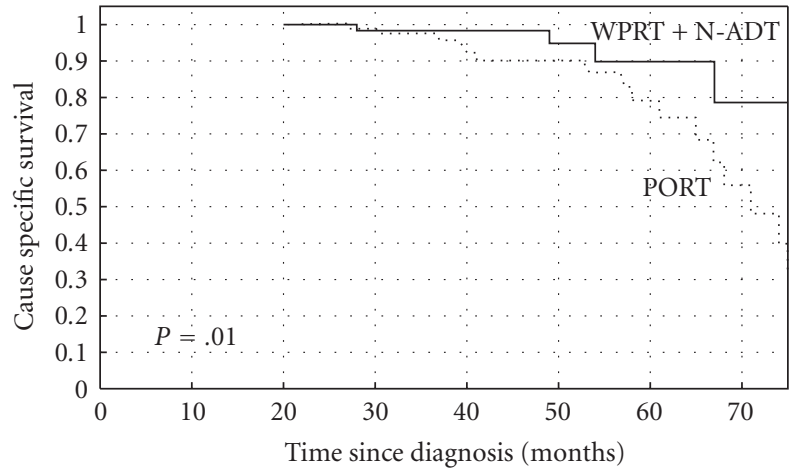

FIgUre 2: The Kaplan-Meier curves comparing cause specific survival (CSS) for patients at high-risk of nodal involvement, as defined in text receiving whole pelvic radiotherapy (WPRT) combined with neoadjuvant androgen deprivation therapy $(\mathrm{N}$ $\mathrm{ADT})$ or prostate radiotherapy only (PORT), $(P=.01)$.

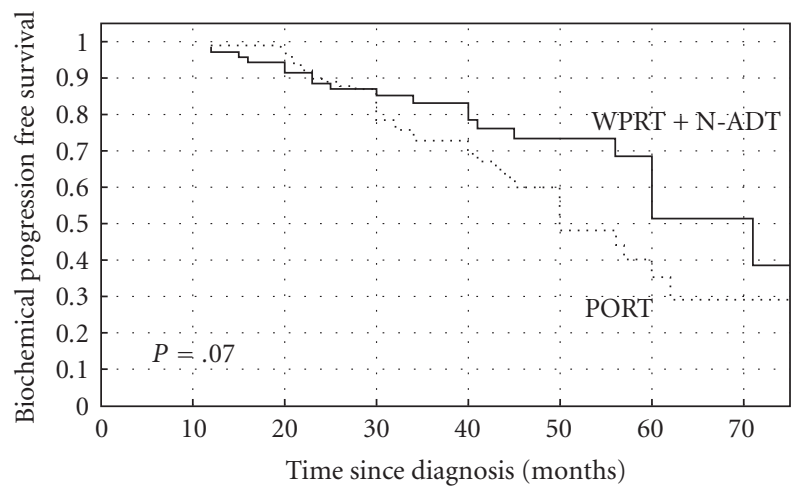

Figure 3: The Kaplan-Meier curves comparing biochemical progression-free survival (bPFS) for patients at high-risk of nodal involvement, as defined in text receiving whole pelvic radiotherapy (WPRT) combined with neoadjuvant androgen deprivation therapy (N-ADT) or prostate radiotherapy only (PORT), $(P=.07)$.

On multivariate analysis combined treatment (WPRT + N-ADT $)(P=.01)$, lower PSA level $(P=.01)$ and lower risk of lymph nodes involvement $(P=.01)$ were statistically significantly associated with longer CSS (Table 3 ).

4.1. Acute and Late Side Effects. Generally, RT in both groups was well tolerated (Table 2). These symptoms typically appeared during the third week of treatment and resolved within a few weeks later. Late side effects grade 2 were noted in minority of patients. No grade 4 toxicities were noted for gastrointestinal and genitourinary tracts.

\section{Discussion}

Our study showed that short N-ADT combined with WPRT and L-ADT provides significant advantage to PORT combined with L-ADT in terms of CSS, DMFS, and bPFS. There was no OS benefit for the WPRT combined with N-ADT in comparison to PORT. Obviously, longer followup is needed to translate the benefit from the CSS

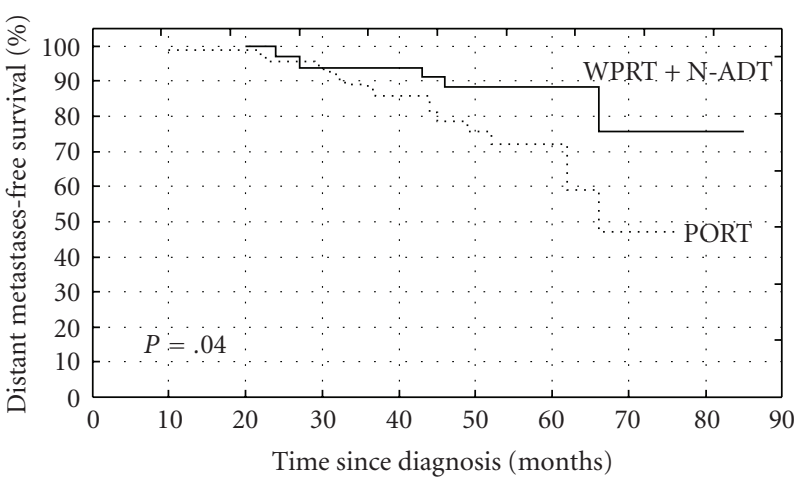

FIgURE 4: The Kaplan-Meier curves comparing distant metastasesfree survival (DMFS) for patients at high-risk of nodal involvement, as defined in text receiving whole pelvic radiotherapy (WPRT) combined with neoadjuvant androgen deprivation therapy $(\mathrm{N}-$ $\mathrm{ADT})$ or prostate radiotherapy only (PORT), $(P=.04)$.

and bPFS to OS. Interestingly, one of the last reviews conducted by Dirix et al. indicated similar conclusions from other studies [23]. At present, conformal radiotherapy is one of the most popular modality of treatment for patients with intermediate and high-risk groups of prostate cancer. According to the American Urology Association (AUA), National Cancer Comprehensive Network (NCCN), National Cancer Institute (NCI), and the European Urology Association (EUA) guidelines the 3D CRT combined with ADT is the most advocated standard of care for patients with high-risk of relapse [24-26]. These guidelines are based on several randomized clinical trials which have demonstrated the benefit of combined therapy (radiotherapy plus ADT) $[27,28]$. However, the optimal timing and duration of hormonal treatment which is combined with radiotherapy remains unresolved [29].

The main limitation of our study is that its nonrandomized trial and thus, it can introduce selection bias. Moreover, while the comparison of study groups (Table 1) showed that they were similar, group A (N-ADT plus WPRT) had marginally more aggressive tumors (more patients with GS 8-10 or T3 tumors, higher PSA, and higher risk of lymph node metastases). Thus, one might generate the hypothesis that the small benefit of WPRT combined with N-ADT would be grater (and difference in OS could be significant) if the study groups were better balanced. Definitely, well designed controlled studies are warranted to check such hypothesis. For example, Wang and Lawton stated that further studies of above mentioned issues are necessary to define the best treatment for patients with high risk of pelvic lymph node involvement [30].

In our study, heterogeneity of therapy modalities used in group A (N-ADT plus WPRT) can blur the final findings of our analysis. Thus, it is impossible to indicate exactly the factor or factors (WPRT only or N-ADT only or WPRT $+\mathrm{N}$ ADT) which were responsible for the achieving better results than those in group B. On the other hand, clinical trial RTOG 94-13 suggested that benefit of WPRT could be achieved if large volume was connected with N-ADT. 
TABLE 2: Acute and late gastrointestinal and genitourinary side effects reported in study for high-risk patients $(n=162)$.

\begin{tabular}{lcc}
\hline Side effects & WPRT + N-ADT (group A) $n=70$ & PORT (group B) $n=92$ \\
\hline Genitourinary acute & $42(60 \%)$ & $50(55 \%)$ \\
Grade 1 & $17(25 \%)$ & $18(21 \%)$ \\
Grade 2 & $2(3 \%)$ & $3(3 \%)$ \\
Grade 3 & & \\
Gastrointestinal acute & $50(71 \%)$ & $48(52 \%)$ \\
Grade 1 & $25(36 \%)$ & $41(44 \%)$ \\
Grade 2 & $45(64 \%)$ & $51(56 \%)$ \\
Grade 3 & & $38(41 \%)$ \\
Genitourinary late & $19(27 \%)$ & $54(59 \%)$ \\
Grade 1 & $51(73 \%)$ & $3(\%)$ \\
Grade 2 & $3(\%)$ & .56 \\
Grade 3 & & $69(52 \%)$ \\
Gastrointestinal late & $51(71 \%)$ & $5(44 \%)$ \\
Grade 1 & $5(36 \%)$ & $3(\%)$ \\
Grade 2 & $2(\%)$ & .088 \\
Grade 3 & 32 \\
\hline
\end{tabular}

WPRT: whole pelvic radiotherapy, PORT: prostate radiotherapy only.

TABLE 3: Univariate and multivariate analysis of clinical and treatment factors predicting for cause specific survival $(n=162)$.

\begin{tabular}{lccc}
\hline Factor & Univariate* & $P$ & Hazard ratio (95\% confidence interval) \\
\hline WPRT + N-ADT versus PORT & .01 & .03 & $2.4(1.5-3.1)$ \\
Age $(<70$ versus $>70)$ & .23 & - & - \\
PSA level $(<30$ versus $>30)$ & .09 & - & - \\
T stage $($ T2c versus T3) & .1 & - & - \\
Gleason score $(2-7$ versus $8-10)$ & .02 & .04 & $1.3(1.08-2.5)$ \\
$\% N+(<30$ versus $>30)$ & .001 & .03 & $2.1(1.4-2.2)$ \\
\hline
\end{tabular}

WPRT: whole pelvic radiotherapy, N-ADT: neoadjuvant androgen deprivation therapy, PORT: prostate radiotherapy only, PSA: prostatic specific antigen, $\% \mathrm{~N}+$ : probability of metastases to pelvic lymph nodes calculated according to the Roach formula [18].

* Kaplan-Meier analysis log-rank $P$-value.

Androgen deprivation therapy could be combined with $\mathrm{RT}$ in many ways, for example, as only N-ADT, or on the other hand, as only L-ADT, or combination of both. In addition, apart from different possibilities of adding ADT and duration of therapy, the differences in irradiated clinical target volume (PORT only or WPRT) may influence the results of therapy. One of the studies which indicated a survival benefit of N-ADT combined with WPRT was RTOG 86-10 trial [31]. In this study, the benefits were limited to a group of patients with bulky disease and Gleason scores of 2 to 6. Reanalysis of conducted RTOG trials indicated that NADT could be beneficial only in a group of patients extended to T1, T2, and Gleason score of 7 . However, there is no clear statement concerning the implementation of N-ADT when radiotherapy is fundamental method of treatment for patients with high risk of relapse. WPRT alone (without ADT) has a weak influence on the results of treatment $[12,14]$. Probably dose in range from $44 \mathrm{~Gy}$ to $50 \mathrm{~Gy}$ is too low to efficiently "sterilize" lymph nodes from cancer cells. In addition, another factor which is responsible for disappointing results of WPRT without N-ADT is high risk of distant metastases when high risk involvement of lymph nodes is noted. It is obvious that for these patients even efficient loco-regional therapy has small impact on distant metastases and in consequence on overall survival. Thus, many investigators in clinical trials are looking for efficient systemic therapy for this group of patients [32]. The simplest and most convenient way of realizing such scenario in clinical practice would be implementation of ADT. For example, the importance of N-ADT combined with RT was investigated by D'Amico et al. in randomized trial where 6month N-ADT was compared with radiotherapy alone [33]. Patients included into this study were classified as a high-risk group. What is worth adding, no pelvic irradiation (WPRT) had been performed. In conclusion, after the median 45months followup, authors stated that significant OS benefit was noted when 6 months of N-ADT were implemented. Another important issue is the duration of N-ADT when it is combined with radiotherapy. Trial performed by D'Amico et al. indicated that 6 month $\mathrm{N}$-ADT is beneficial. Is there any difference in effectiveness when short N-ADT (3 months) is combined with radiotherapy? The Canadian trial which 
compares short-term N-ADT (3 months) with long-term N-ADT (8 months) [34] showed that longer ADT has no advantage over short therapy in the high-risk patients. In our study the median time of N-ADT was 4.4 months and was similar to proposed in majority of clinical trials. Similarly, benefit of short-term ADT, in particular in high-risk patients, was demonstrated in the EORTC study conducted by Bolla et al., Denham et al., and in the Australian study $[35,36]$.

Another very important issue is evaluation of the role of L-ADT when N-ADT is combined with radiotherapy. In literature there are many well prepared clinical studies which proved that long-term adjuvant ADT is more efficient than PORT alone. One of the examples is study conducted by RTOG (RTOG 9202) where N-ADT was introduced 2 months prior to irradiation (short term of N-ADT) and then was continued during radiotherapy [6]. In the investigational arm the patients were treated with ADT for 2 years after the end of radiotherapy. In a group of patients treated with LADT and radiotherapy the 5-year OS rate was $80 \%$, while in the group of patients treated without additional hormonal therapy was 69\%, respectively. Another well documented study, which showed a therapeutic benefit, had been carried out by Bolla et al. who confirmed that L-ADT conducted for 3 years resulted in an increase of 5 years OS from $62 \%$ to $79 \%$ $(P=.001)$ [27]. However, ADT combined with radiotherapy in high-risk group of patients gives poor results.

In our study L-ADT (28 months) was added in both treatment groups. ADT duration was similar to reported by these two mentioned trials. Interestingly, all patients included into these trials were treated with the WPRT. In trial 9202 RTOG the short N-ADT and WPRT were implemented. On the other hand, none of the mentioned trials included the arm with PORT combined with the L-ADT. As a result, these trials could not test the role of WPRT combined with NADT and L-ADT when in comparison arm was not included PORT with L-ADT. RTOG 9202 study indicated that L-ADT is better strategy of treatment for high-risk patients than short-term N-ADT. However, this study did not evaluate the role of WPRT on the results of treatment.

One of the crucial clinical trials which aim is to answer the question concerning the role of WPRT combined with N-ADT is RTOG $9413[10,11,15]$. This is a four-arm trial which is trying to evaluate the role of WPRT combined with short N-ADT or short adjuvant ADT (4 months). The early results indicated that implementation of WPRT and short N-ADT had no advantage over PORT plus N-ADT and short-adjuvant ADT or PORT plus short-adjuvant ADT. After followup of 44 months there was no benefit in OS, but the results defined as CSS, DMFS, bPFS were significantly better for WPRT combined with N-ADT. Comparison of all four arms indicated that a clear benefit for patients treated with WPRT and N-ADT (4-year disease-free survival of $60 \%$ ) was over all three other arms (44\% versus $49 \%$ versus 50\%, resp.; $P=.008$ ). RTOG 9413 study specifically addressed the issue of treatment volume and demonstrated a sequence dependent benefit associated with WPRT and NADT. This trial demonstrated that when N-ADT is applied in conjunction with irradiation (WPRT) it yields a better progression disease-free survival than PORT even for low dose of irradiation ( $46 \mathrm{~Gy}$ ) to the pelvis. Comparison of this trial with our study indicated the differences in the applied L-ADT. In our study, the L-ADT (28 months) had been implemented for both arms (PORT and WPRT). Our study in this aspect was similar to RTOG 9202 and EORTC 22863. This is clear and very important statement, because in the trial RTOG 9413 only short (4 months) adjuvant ADT was implemented. Results of RTOG 9413 and our study suggested that the addition of ADT before starting of irradiation could be regarded as the additional biological dose, which kills some number of clonogenic cancer cells and creates better treatment conditions for further radiotherapy. Of course, the decreasing level of PSA after N-ADT cannot be interpreted as proportional diminishing of the number of cancer cells. It should be underlined that the latest update of trial 8610 stated in conclusion that the addition of 4 months of N-ADT to radiotherapy has a dramatic impact on clinically meaningful end points in men with locally advanced disease [37]. The most important clinical trials in which hormonal therapy has been combined with radiotherapy were implementing WPRT.

One of the most important issues of our study is the early and late toxicities. Our results indicated that the level of toxicities was acceptable. Although, according to our observations in the group treated with the N-ADT and WPRT the intensity of early and late toxicities was more pronounced. However, N-ADT caused the significant reduction in the volume of irradiated organs at risk because of reduced volume either of prostate cancer or volume of the enlarged prostate gland. The median reduction of target volume observed by Zelefsky et al. was 25\% [38]. On the other hand, in the first phase of irradiation (WPRT) more healthy tissue was irradiated. When aiming to minimize the side effects it is important to introduce the N-ADT 2-3 months before starting of irradiation. This policy was mandatory because a decrease in the volume of the prostate gland during the course of irradiation may result in position changes of the organs at risk and could shift them into volume of higher irradiation dose.

\section{Conclusions}

Our results showed that WPRT combined with N-ADT and L-ADT compared to PORT combined with N-ADT and LADT had an advantage for CSS and bPFS. WPRT was well tolerated with modest acute and late gastrointestinal and genitourinary toxicity. It is obvious that N-ADT plus WPRT could be standard of care for high-risk patients, but question concerned the gain in OS is still open.

\section{References}

[1] F. Bray, R. Sankila, J. Ferlay, and D. M Parkin, "Estimates of cancer incidence and mortality in Europe in 1995," European Journal of Cancer, vol. 38, no. 1, pp. 99-166, 2002.

[2] R. J. Black, F. Bray, J. Ferlay, and D. M. Parkin, "Cancer incidence and mortality in the European union: cancer registry data and estimates of national incidence for 1990," European Journal of Cancer Part A, vol. 33, no. 7, pp. 10751107, 1997. 
[3] R. T. Greenlee, M. B. Hill-Harmon, T. Murray, and M. Thun, "Cancer statistics, 2001," CA: A Cancer Journal for Clinicians, vol. 51, no. 1, pp. 15-36, 2001.

[4] G. D. Grossfeld, J. J. Chang, J. M. Broering, et al., "Impact of positive surgical margins on prostate cancer recurrence and the use of secondary cancer treatment: data from the CaPSURE database," Journal of Urology, vol. 163, no. 4, pp. 1171-1177, 2000.

[5] A. L. Zietman, "Dose escalation in localized prostate cancer: make no assumptions," International Journal of Radiation Oncology Biology Physics, vol. 54, no. 3, pp. 635-636, 2002.

[6] G. E. Hanks, T. F. Pajak, A. Porter, et al., "Phase III trial of long-term adjuvant androgen deprivation after neoadjuvant hormonal cytoreduction and radiotherapy in locally advanced carcinoma of the prostate: the Radiation Therapy Oncology Group Protocol 92-02," Journal of Clinical Oncology, vol. 21, no. 21, pp. 3972-3978, 2003.

[7] J. Laverdière, J. L. Gomez, L. Cusan, et al., "Beneficial effect of combination hormonal therapy administered prior and following external beam radiation therapy in localized prostate cancer," International Journal of Radiation Oncology Biology Physics, vol. 37, no. 2, pp. 247-252, 1997.

[8] M. Roach III, J. Lu, M. V. Pilepich, et al., "Predicting longterm survival, and the need for hormonal therapy: a metaanalysis of RTOG prostate cancer trials," International Journal of Radiation Oncology Biology Physics, vol. 47, no. 3, pp. 617627, 2000.

[9] M. V. Pilepich, K. Winter, C. A. Lawton, et al., "Androgen suppression adjuvant to definitive radiotherapy in prostate carcinoma-long-term results of phase III RTOG 85-31," International Journal of Radiation Oncology Biology Physics, vol. 61, no. 5, pp. 1285-1290, 2005.

[10] M. Roach III, M. DeSilvio, C. Lawton, et al., "Phase III trial comparing whole-pelvic versus prostate-only radiotherapy and neoadjuvant versus adjuvant combined androgen suppression: Radiation Therapy Oncology Group 9413," Journal of Clinical Oncology, vol. 21, no. 10, pp. 1904-1911, 2003.

[11] M. Roach III, M. DeSilvio, R. Valicenti, et al., "Wholepelvis, "mini-pelvis," or prostate-only external beam radiotherapy after neoadjuvant and concurrent hormonal therapy in patients treated in the Radiation Therapy Oncology Group 9413 trial," International Journal of Radiation Oncology Biology Physics, vol. 66, no. 3, pp. 647-653, 2006.

[12] C. E. Vargas, R. Galalae, J. Demanes, et al., "Lack of benefit of pelvic radiation in prostate cancer patients with a high risk of positive pelvic lymph nodes treated with high-dose radiation," International Journal of Radiation Oncology Biology Physics, vol. 63, no. 5, pp. 1474-1482, 2005.

[13] A. Zapatero, F. Garcia-Vicente, I. Modolell, et al., "Impact of mean rectal dose on late rectal bleeding after conformal radiotherapy for prostate cancer: dose-volume effect," International Journal of Radiation Oncology Biology Physics, vol. 59, no. 5, pp. 1343-1351, 2004.

[14] S. S. Ploysongsang, B. S. Aron, and W. M. Shehata, "Radiation therapy in prostate cancer: whole pelvis with prostate boost or small field to prostate?" Urology, vol. 40, no. 1, pp. 18-26, 1992.

[15] C. A. Lawton, M. DeSilvio, M. Roach III, et al., "An update of the phase III trial comparing whole pelvic to prostate only radiotherapy and neoadjuvant to adjuvant total androgen suppression: updated analysis of RTOG 94-13, with emphasis on unexpected hormone/radiation interactions," International Journal of Radiation Oncology Biology Physics, vol. 69, no. 3, pp. 646-655, 2007.
[16] S. A. Seaward, V. Weinberg, P. Lewis, B. Leigh, T. L. Phillips, and M. Roach III, "Improved freedom from PSA failure with whole pelvic irradiation for high-risk prostate cancer," International Journal of Radiation Oncology Biology Physics, vol. 42, no. 5, pp. 1055-1062, 1998.

[17] S. A. Seaward, V. Weinberg, P. Lewis, B. Leigh, T. L. Phillips, and M. Roach III, "Identification of a high-risk clinically localized prostate cancer subgroup receiving maximum benefit from whole-pelvic irradiation," Cancer Journal from Scientific American, vol. 4, no. 6, pp. 370-377, 1998.

[18] M. Roach III, C. Marquez, H.-S. Yuo, et al., "Predicting the risk of lymph node involvement using the pre-treatment prostate specific antigen and Gleason Score in men with clinically localized prostate cancer," International Journal of Radiation Oncology Biology Physics, vol. 28, no. 1, pp. 33-37, 1994.

[19] M. Roach III, G. Hanks, H. Thames Jr., et al., "Defining biochemical failure following radiotherapy with or without hormonal therapy in men with clinically localized prostate cancer: recommendations of the RTOG-ASTRO Phoenix Consensus Conference," International Journal of Radiation Oncology Biology Physics, vol. 65, no. 4, pp. 965-974, 2006.

[20] P. Milecki, T. Piotrowski, and M. Dymnicka, "The comparison of radiotherapy techniques for treatment of the prostate cancer: the three-field vs. the four-field," Neoplasma, vol. 51, no. 1, pp. 64-69, 2004.

[21] J. D. Cox, J. Stetz, and T. F. Pajak, "Toxicity criteria of the Radiation Therapy Oncology Group (RTOG) and the European Organization for Research and Treatment of Cancer (EORTC)," International Journal of Radiation Oncology Biology Physics, vol. 31, no. 5, pp. 1341-1346, 1995.

[22] American Society for Therapeutic Radiology, Oncology Consensus Panel, "Consensus statement: guidelines for PSA following radiation therapy," International Journal of Radiation Oncology Biology Physics, vol. 37, no. 5, pp. 1035-1041, 1997.

[23] P. Dirix, K. Haustermans, S. Junius, R. Withers, R. Oyen, and H. Van Poppel, "The role of whole pelvic radiotherapy in locally advanced prostate cancer," Radiotherapy and Oncology, vol. 79, no. 1, pp. 1-14, 2006.

[24] American Urology Association Guidelines for Prostate Cancer, http://www.auanet.org.

[25] European Urology Association Guidelines for Prostate Cancer, http://www.uroweb.org.

[26] National Cancer Comprehensive Network .Prostate Cancer, v.2, 2008, http://www.nccn.org.

[27] M. Bolla, D. Gonzalez, P. Warde, et al., "Improved survival in patients with locally advanced prostate cancer treated with radiotherapy and goserelin," The New England Journal of Medicine, vol. 337, no. 5, pp. 295-300, 1997.

[28] M. V. Pilepich, K. Winter, C. A. Lawton, et al., "Androgen suppression adjuvant to definitive radiotherapy in prostate carcinoma-long-term results of phase III RTOG 85-31," International Journal of Radiation Oncology Biology Physics, vol. 61, no. 5, pp. 1285-1290, 2005.

[29] P. Milecki, Z. Kwias, and D. J. Martenka, "Radiotherapy combined with hormonal therapy (RT-HT) in prostate cancer patients with low, intermediate, and high risk of biochemical recurrence: perspective and therapeutic gain analysis," Neoplasma, vol. 54, no. 1, pp. 7-15, 2007.

[30] D. Wang and C. Lawton, "Pelvic lymph node irradiation for prostate cancer: who, why, and when?" Seminars in Radiation Oncology, vol. 18, no. 1, pp. 35-40, 2008.

[31] M. V. Pilepich, K. Winter, M. J. John, et al., "Phase III radiation therapy oncology group (RTOG) trial 86-10 of androgen deprivation adjuvant to definitive radiotherapy in locally 
advanced carcinoma of the prostate," International Journal of Radiation Oncology Biology Physics, vol. 50, no. 5, pp. 12431252, 2001.

[32] J. Friedman, R. L. Dunn, D. Wood, et al., "Neoadjuvant docetaxel and capecitabine in patients with high risk prostate cancer," Journal of Urology, vol. 179, no. 3, pp. 911-916, 2008.

[33] A. V. D’Amico, J. Manola, M. Loffredo, A. A. Renshaw, A. DellaCroce, and P. W. Kantoff, "6-month androgen suppression plus radiation therapy vs radiation therapy alone for patients with clinically localized prostate cancer: a randomized controlled trial," Journal of the American Medical Association, vol. 292, no. 7, pp. 821-827, 2004.

[34] J. Crook, C. Ludgate, S. Malone, et al., "Report of a multicenter Canadian phase III randomized trial of 3 months vs. 8 months neoadjuvant androgen deprivation before standarddose radiotherapy for clinically localized prostate cancer," International Journal of Radiation Oncology Biology Physics, vol. 60 , no. 1, pp. 15-23, 2004.

[35] M. Bolla, L. Collette, L. Blank, et al., "Long-term results with immediate androgen suppression and external irradiation in patients with locally advanced prostate cancer (an EORTC study): a phase III randomised trial," The Lancet, vol. 360, no. 9327, pp. 103-108, 2002.

[36] J. W. Denham, A. Steigler, D. S. Lamb, et al., "Short-term androgen deprivation and radiotherapy for locally advanced prostate cancer: results from the Trans-Tasman Radiation Oncology Group 96.01 randomised controlled trial," Lancet Oncology, vol. 6, no. 11, pp. 841-850, 2005.

[37] M. Roach III, K. Bae, J. Speight, et al., "Short-term neoadjuvant androgen deprivation therapy and external-beam radiotherapy for locally advanced prostate cancer: long-term results of RTOG 8610," Journal of Clinical Oncology, vol. 26, no. 4, pp. 585-591, 2008.

[38] M. J. Zelefsky, S. A. Leibel, C. M. Burman, et al., "Neoadjuvant hormonal therapy improves the therapeutic ratio in patients with bulky prostatic cancer treated with three-dimensional conformal radiation therapy," International Journal of Radiation Oncology Biology Physics, vol. 29, no. 4, pp. 755-761, 1994. 


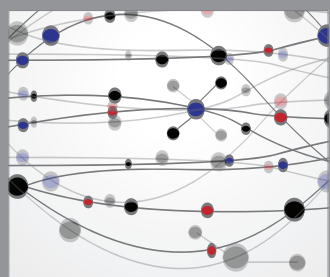

The Scientific World Journal
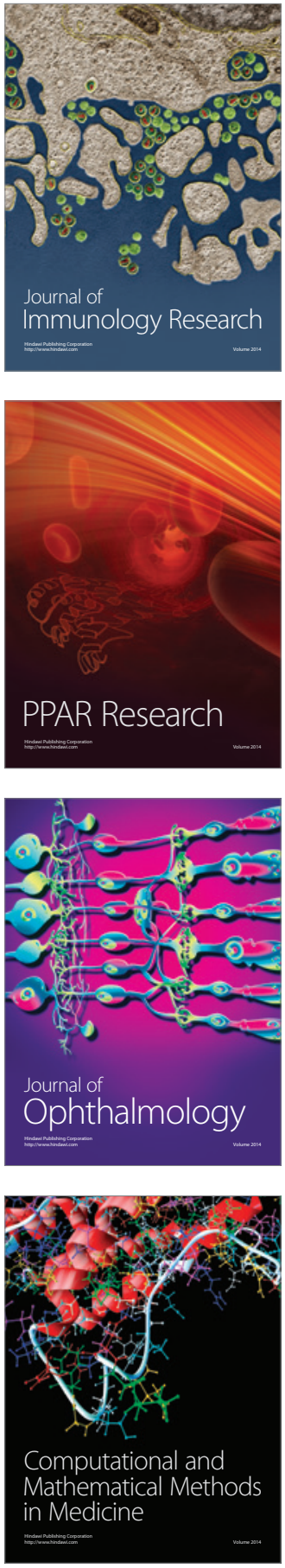

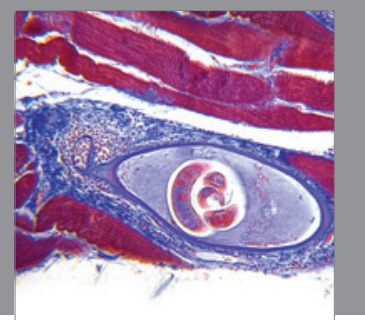

Gastroenterology

Research and Practice
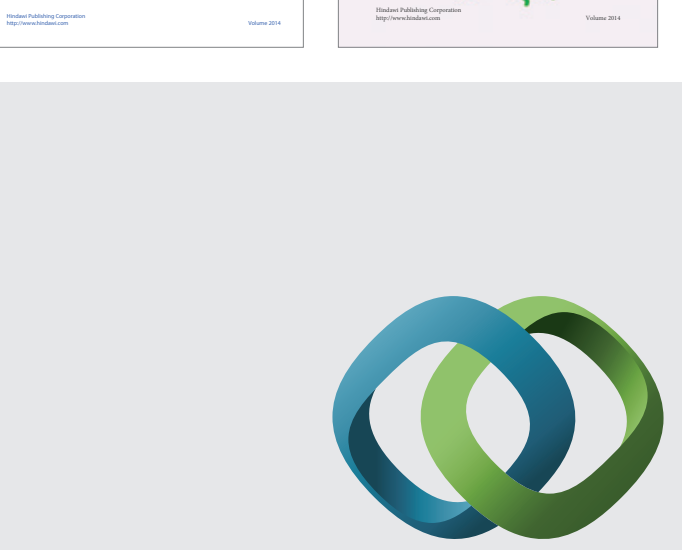

\section{Hindawi}

Submit your manuscripts at

http://www.hindawi.com
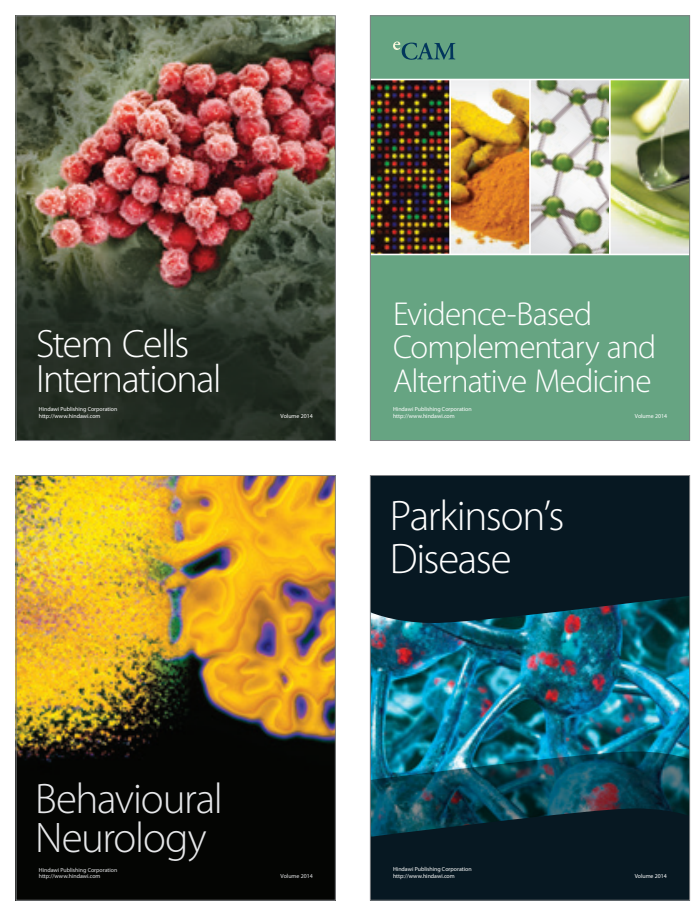

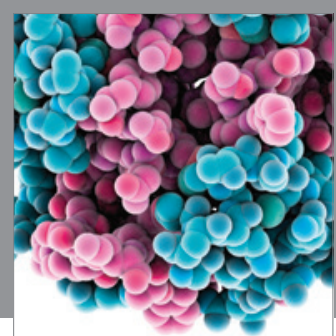

Journal of
Diabetes Research

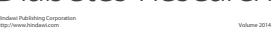

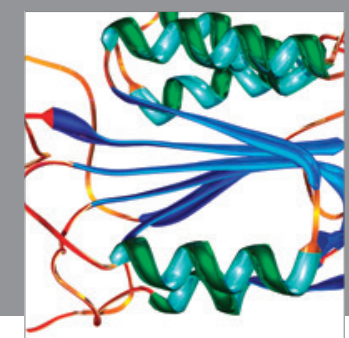

Disease Markers
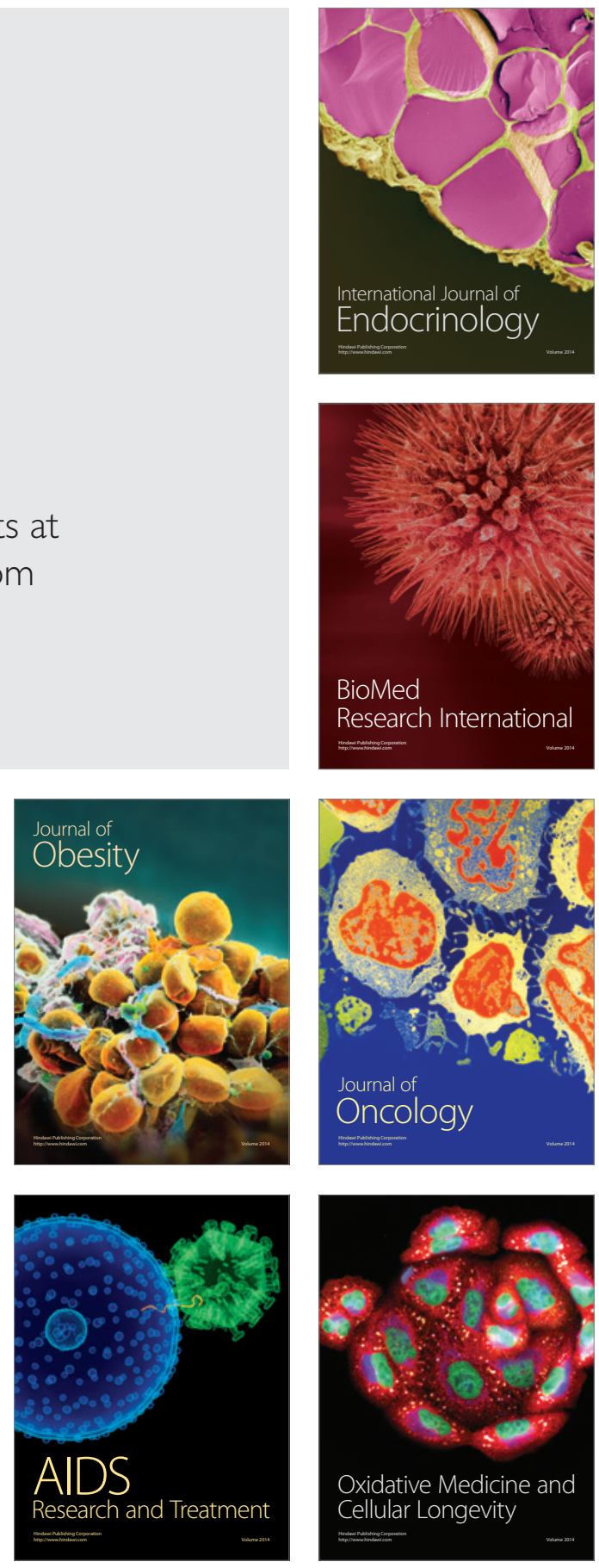